\title{
Gradhiva
}

Revue d'anthropologie et d'histoire des arts

$14 \mid 2011$

Carl Einstein et les primitivismes

\section{Un ensevelissement sous les feuilles}

Bebuquin II, l'autobiographie de Carl Einstein

A Burial in Leaves : Bebuquin II, Carl Einstein's Autobiography

\section{Devin Fore}

Traducteur : Camille Joseph

\section{OpenEdition}

\section{Journals}

Édition électronique

URL : http://journals.openedition.org/gradhiva/2190

DOI : 10.4000/gradhiva.2190

ISSN : 1760-849X

\section{Éditeur}

Musée du quai Branly Jacques Chirac

\section{Édition imprimée}

Date de publication : 30 novembre 2011

Pagination : 164-183

ISBN : 978-2-35744-046-3

ISSN : 0764-8928

Référence électronique

Devin Fore, «Un ensevelissement sous les feuilles », Gradhiva [En ligne], 14 | 2011, mis en ligne le 30 novembre 2014, consulté le 30 avril 2019. URL : http://journals.openedition.org/gradhiva/2190 ; DOI : 10.4000/gradhiva.2190 


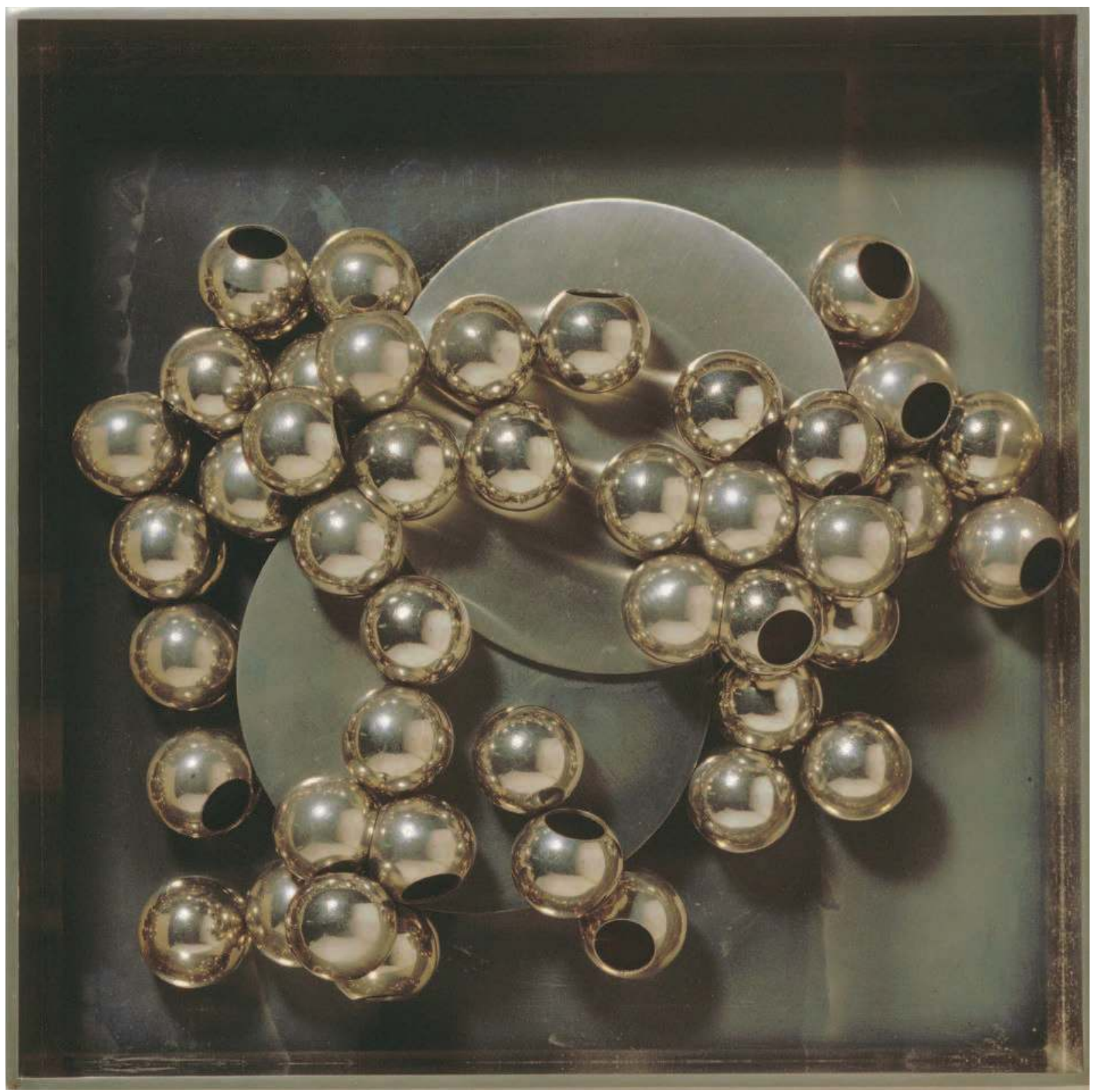

Fig. 1 Alice Hutchins, Flux Box de la série Circuit Box, 1973, collection Centre Pompidou, dist. RMN / Philippe Migeat, DR. 


\section{Un \\ ensevelissement sous les feuilles Bebuquin II, l'autobiographie de Carl Einstein}

Devin Fore
Vers 1925, Carl Einstein se lança dans la rédaction d'une autobiographie qui fut retrouvée inachevée après son suicide en 1940. Il intitula le projet Bebuquin II en référence à son court roman de 1912, Bebuquin oder die Dilettanten des Wunders (Bebuquin ou les dilettantes du miracle), qui l'avait fait connaître comme écrivain moderniste. Le choix du titre de l'autobiographie semble avoir procédé d'une double motivation. D'abord, la référence au célèbre roman expressionniste laisse entendre qu'Einstein comptait sur ce premier succès éditorial pour assurer son grand retour sur la scène littéraire. Depuis la publication de La Sculpture nègre en 1915, Einstein s'était en effet exclusivement consacré à la critique d'art, ce qui avait eu pour effet de rejeter dans l'ombre sa réputation d'auteur littéraire. Les lettres dans lesquelles Einstein évoque son projet d'autobiographie montrent qu'il espérait, grâce à Bebuquin II, inverser cette tendance.

Le retour d'Einstein à Bebuquin dans les années 1920 était en outre motivé par le désir de réécrire son monument moderniste des débuts. À ce titre, Bebuquin II est une expression typique du moment historique qui le vit naître; l'intervalle des années 1920 et 1930, temps de retour sur le passé où l'héritage du modernisme expérimental fut revisité dans le but non de le restaurer mais, au contraire, de rejeter ses modèles artistiques. Dans Bebuquin II, Einstein réussit à révoquer le modernisme en insérant la nouvelle d'origine dans le cadre d'une autobiographie, genre littéraire qui privilégie la fonction dénotative de la langue et qui subordonne le discours littéraire stylisé à une réalité extratextuelle entendue dans un sens emphatique. Dans l'autobiographie, le fait l'emporte sur la métaphore comme référent irrécusable et infaillible de l'écriture. La stratégie visant à replacer et, par suite, à contenir la littérature moderniste dans le cadre de référence des mémoires range Bebuquin II sous la bannière de la Nouvelle Objectivité, un mouvement dont la branche littéraire exprima sa quintessence dans le genre documentaire, notamment avec le reportage ou l'autobiographie. Ces genres, qui 
articulent la dynamique du sens textuel à une référence exophorique, reflètent ce qu'on appelle souvent l'«esthétique de stabilisation » de la Nouvelle Objectivité. De ce point de vue, l'explosion du nombre d'autobiographies et des autres formes documentaires dans les années 1920 et 1930 représentait une réaction culturelle face à l'insécurité matérielle et l'instabilité sociale qui caractérisèrent les années de l'entre-deux-guerres. Ces textes abandonnèrent l'autoréflexivité analytique et les questionnements sémiotiques associés aux expériences littéraires du modernisme pour se placer sur le terrain solide de la réalité objective et de l'expérience dite authentique. Cet élan positiviste trouva son expression la plus achevée dans ce que Leo Löwenthal a désigné, en référence à l'histoire de la Nouvelle Objectivité, comme une "sorte d'anthropologie fossilisée » (eine Art versteinerter Anthropologie ${ }^{1}$.

Le projet Bebuquin II d'Einstein est l'un des textes qui illustre le mieux cette évolution. Il est loin d'afficher la même exubérance linguistique que son prédécesseur, Bebuquin. Le ton y est au contraire détaché et flegmatique. Là où l'écriture faisait auparavant la part belle à l'ouverture et au doute, les énigmes herméneutiques ont désormais disparu. Bebuquin II répudie l'autoréférentialité du modernisme littéraire et se met en quête d'une langue qui puisse répondre à la réalité, une langue de la conséquence et de la responsabilité politique. Dans ce texte, Einstein exprime sa méfiance à l'égard de la tendance solipsiste de la littérature moderne en développant une critique cinglante de la fictionnalité elle-même, dans laquelle il désavoue l'imagination artistique et exige de remplacer la spéculation abstraite par l'action politique directe. Associée à son pendant théorique, la grande tirade antimoderniste intitulée La Fabrication des fictions, Bebuquin II anticipait le verdict final sur la littérature qu'Einstein prononça en 1936 : après avoir rejoint cette année-là la milice anarchiste de Buenaventura Durruti en Espagne, il ne publia plus jamais d'œuvre littéraire.

Bebuquin II mérite sans nul doute le qualificatif d'œuvre réaliste si l'on s'en tient au niveau de la phrase individuelle, où Einstein parvient à contenir la syntaxe explosive et à limiter les ambiguïtés lexicales du premier Bebuquin. Cependant, le programme réaliste est moins évident à l'échelle plus large des dynamiques textuelles. Prises séparément, les plus petites unités du texte font parfaitement sens, mais, une fois réunies, elles produisent une sorte d'alchimie herméneutique. Il ne s'agit pas de dire ici que ces unités textuelles ne s'accordent pas entre elles : au contraire, elles s'accordent trop bien. Le lecteur fait ainsi l'expérience immédiate d'un texte clair et sobre, mais un courant souterrain va simultanément à l'encontre de l'intelligibilité des scènes individuelles, et son impact s'accroît au fur et à mesure que se succèdent les 1300 unités textuelles isolées qui composent l'autobiographie. Par exemple, Einstein répète à plusieurs reprises un épisode de sa vie, qu'il fait rejouer par une constellation nouvelle de personnages et dont, par conséquent, la fin varie à chaque fois. Ces différentes conjugaisons narratives confèrent à l'architecture temporelle de Bebuquin II une qualité mystérieusement circulaire, qui est renforcée par la tendance du narrateur à naviguer entre l'ici et maintenant du journal et l'usage du prétérit révolu de l'épopée. À ces sauts entre les temps du présent et du passé s'ajoutent des passages de la première à la troisième personne. L'usage de la troisième personne installe une perspective extérieure qui leste l'autobiographie en lui conférant un sens objectif de réalité historique; pourtant, par moments, cette même extériorité renvoie à un état de dissociation mentale extrême, comme si Einstein s'observait de l'extérieur. En rompant avec l'équilibre délicat entre l'enregistrement objectif des faits 


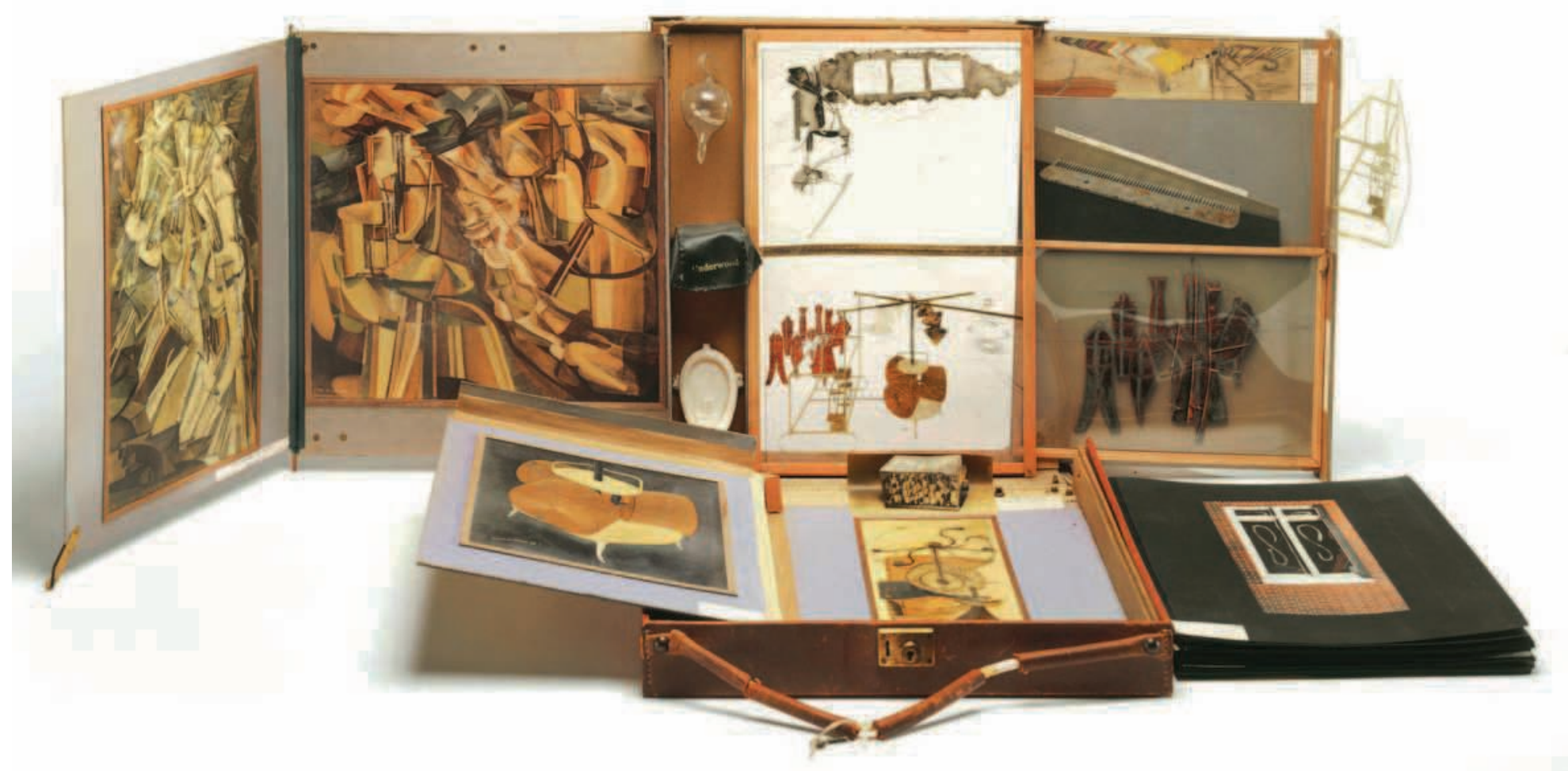

Fig. 2 Marcel Duchamp, La Boite-en-valise, 1936, collection Centre Pompidou, dist. RMN / Philippe Migeat @ Succession Marcel Duchamp / ADAGP, Paris 2011.

et l'intériorité psychologique propre au genre des mémoires, Bebuquin II dévoile une loi de l'écriture autobiographique : le document du moi ne peut tolérer l'objectivité et l'extériorité que jusqu'à un certain seuil, au-delà duquel son sens de la réalité tombe dans l'excès inverse, à savoir la déréalisation psychique complète. Dans ces moments-là, au lieu d'ancrer ontologiquement la langue dans des référents extérieurs au texte, Bebuquin II succombe à une paramnésie confuse qui ébranle la confiance du lecteur dans l'existence d'une vérité extratextuelle absolue. Ajoutons ici que cette boucle rétroactive entre Bebuquin II et la vie de son auteur affecta peu à peu la capacité d'Einstein à distinguer entre les deux. Loin de renforcer la frontière entre écriture et réalité, Bebuquin II revêtit un tour hémorragique et déborda dans la vie d'Einstein pendant les années d'écriture du projet, l'auteur se mettant même à signer ses lettres de la lettre $B$, en référence à son protagoniste.

Les scènes et les épisodes de Bebuquin II renvoient ainsi l'image d'un texte réaliste, alors que, du point de vue du registre métatextuel, l'impression est très différente : l'autobiographie laisse entrevoir une radicalisation du programme de l'avant-garde plutôt que son abrogation. Dans le cas d'Einstein, on peut dès lors difficilement concevoir l'autobiographie comme « un moyen de mettre à l'abri son identité »; Bebuquin II ne propose aucun récit de Bildung ou de construction de soi (self-fashioning). Bien au contraire. Ici, l'acte de remémoration devient un moyen de tailler en pièces le moi dans un autodafé textuel. Ce livre, qu'Einstein pensa également appeler Verreck (Crève!), était un «nécrologe du moi" ". "Je
- $\bullet$

2. Archives Carl Einstein, Akademie der Künste Berlin, dossiers 58, 1 ?. Dans la suite, la référence Archives Carl Einstein sera placée dans le texte et suivie du numéro de dossier. 
mènerai une révolte contre le moi ", écrivait-il dans ses notes en vue de ce projet, "je dois disparaître» (Archives Carl Einstein 17). Influencé par la psychologie néo-associationniste de son temps, Einstein concevait le moi comme un édifice construit à partir de traces de mémoire : il était « rétrospectif, une exhibition de souvenirs ${ }^{3}$. Einstein estimait que le moi, parce qu'il se définissait et se stabilisait dans la continuité avec le passé, représentait une construction psychique fondamentalement passéiste : l'accès au présent et l'éventualité d'un changement futur se trouvaient ainsi forclos. Le moi, qui maintenait le sujet sous le joug du passé, fonctionnait comme une sclérose mnémonique interdisant des expériences nouvelles. Einstein espérait alors que le démantèlement de l'architecture du moi, de la structure psychique qui s'était considérablement renforcée à l'époque de l'individualisme bourgeois, ouvrirait la voie à une rencontre plus directe avec le monde, affranchie de la congestion névrotique. La destruction du moi dans l'enquête autobiographique était le premier pas vers la restauration d'un métabolisme épistémologique ouvert entre le sujet et le monde.

Mais une aporie se fait ainsi jour d'emblée dans la logique opérationnelle de Bebuquin II. Pourquoi Einstein, qui recherchait une technique d'éradication du moi, choisit-il le genre de l'autobiographie - entrepôt de la mémoire et temple littéraire de l'ego - pour partir en guerre contre le sujet psychologique? Puisque l'objectif était d'exercer une critique du moi, il aurait été plus logique de fuir le locus textuel de l'ego plutôt que de l'investir. Comment un projet d'autoprospection pouvait-il espérer déjouer les conditions de l'individualité, dont il présupposait qu'elles formaient le point de départ? La stratégie déployée par Einstein était profondément dialectique et faisait converger une «hypertrophie de la personnalité » autobiographique (Archives Carl Einstein 14) et son contraire, à savoir un "nécrologe du moi ». Einstein conçut Bebuquin II comme un texte destiné à faire éclater les composantes de la subjectivité au lieu de les relier les unes aux autres, une anamnèse appelée à détruire le contenu de la mémoire par l'acte même de remémoration. L'éradication du noyau psychologique de l'individualité ne serait pas obtenue par la négation du moi, mais par un excès de moi et une surabondance de souvenirs. Cette économie mnémonique s'exprimait dans la définition troublante donnée par Einstein de l'entreprise autobiographique comme «oubli égoïste du moi " (Archives Carl Einstein 19).

Le projet de démantèlement de la faculté de mémoire et de l'autorité du moi rejoint une critique plus générale du réalisme mimétique. Les essais critiques d'Einstein sur la peinture cubiste française fournissent un premier aperçu de la manière dont il concevait la collusion entre mémoire et réalisme de la représentation. Dans un article publié par la revue Documents sur le cubisme analytique, la phase de production cubiste qui s'étendit de 1909 à 1914, Einstein affirmait que la grande innovation du cubisme ne résidait pas dans la rupture avec l'optique unifiée et cohérente du spectateur, ni dans le coup porté par cette fragmentation visuelle à l'intégrité du sujet spectateur. Ces effets étaient secondaires par rapport à la vocation première du cubisme, à savoir la subversion de la ressemblance mimétique par la destruction de la mémoire. Einstein expliquait dans ce texte que la vraisemblance optique reposait sur la répétition de signes conventionnels qui déclenchaient les images tactiles et tirées de l'expérience conservées dans la mémoire du spectateur. Dans la rencontre

3. Carl Einstein in Rolf-Peter Baacke (éd.), Werke, t. III, Berlin, Medusa, 1980-1996, p. 118. Désormais cité dans le texte sous la référence Werke. avec un tableau, la façon dont le spectateur déchiffrait l'image dépendait de sa capacité à puiser dans une réserve mentale de représentations préexistantes dont il se servait pour établir des comparaisons. De cette manière, l'«art des- 


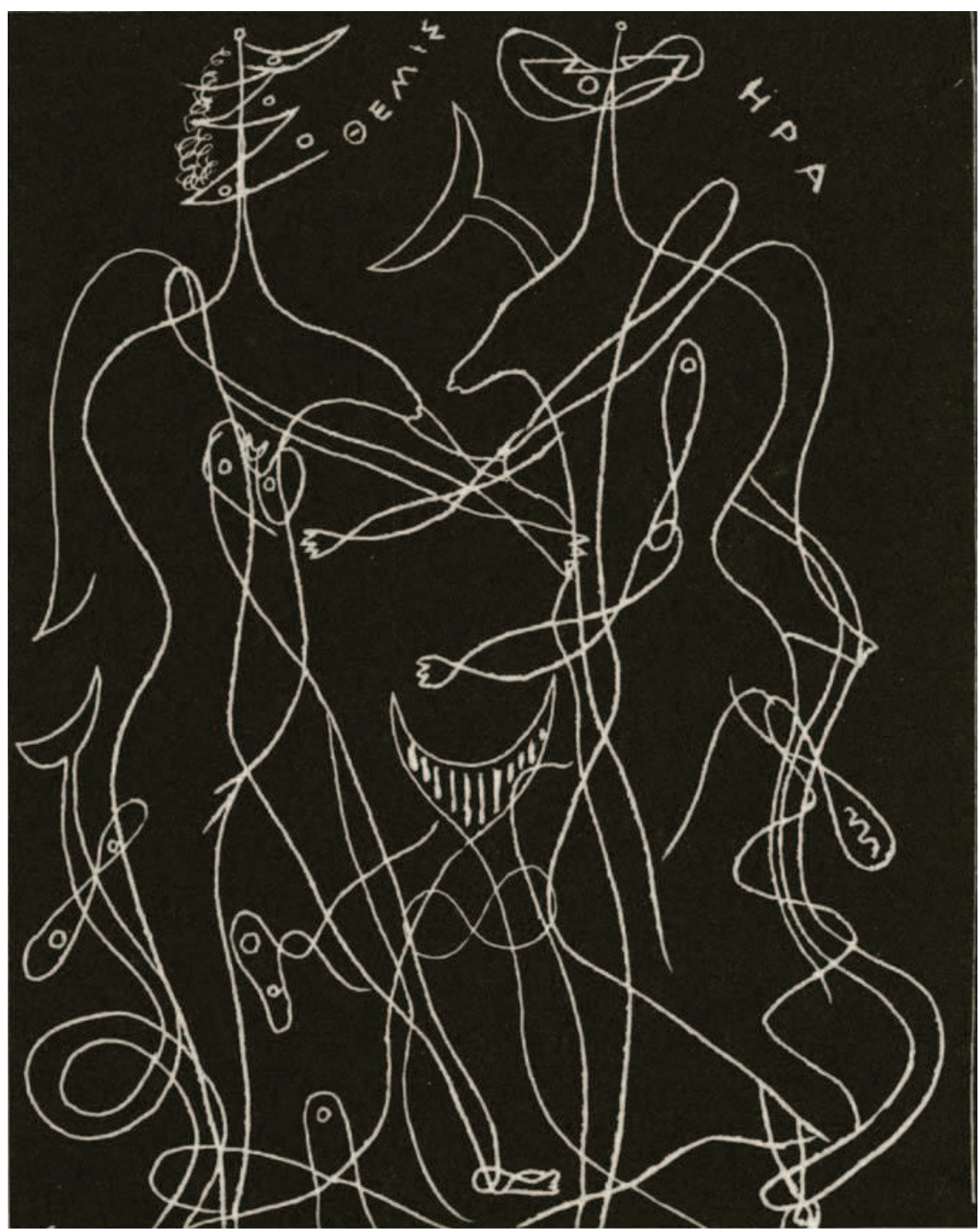

Fig. 3 Georges Braque, Themis et Hera, 1934 @ ADAGP, Paris 2011.

criptif » - terme sous lequel Einstein désignait le réalisme mimétique - croisait l'objet perçu avec un "dépôt de mémoire» (Einstein, Werke IV : 163). Mais les cubistes analytiques, poursuivait Einstein, avaient rompu le lien entre perception optique et mémoire, et transgressé ainsi le «besoin d'identité » du réalisme mimétique : «Ce furent les cubistes qui ébranlèrent l'objet toujours identique à lui-même, c'est-à-dire la mémoire dans laquelle les notions sont adaptées les unes aux autres. Leur mérite principal est d'avoir détruit les images mnémoniques. [...] Il fallut détruire l'héritage mnémonique des objets, c'est-à-dire oublier, et le tableau devint non la fiction d'une autre réalité, mais une réalité avec ses propres conditions. " (Einstein, Werke III : 488) Le programme du cubisme analytique, observait Einstein, cherchait à anéantir la ressemblance en radicalisant une mesure d'oubli forcé qui restaurait la présence de l'objet esthétique. Cet oubli était obtenu par la négation de la profondeur picturale, qui focalisait l'attention sur la surface de la toile et la facture matérielle du tableau. «La condition 
première est la surface. On ne travaille plus entre deux couches imaginaires qui dépassent la toile. Maintenant, la totalisation du tableau s'opère par son invérifiabilité, et le fait que le spectateur ne sort pas de la réalité du tableau. " (Ibid. : 489) [fig. 4] Dans la tension entre l'absence sémiotique et la présence phénoménologique, le tableau de Georges Braque oscille entre le signe visuel d'un torse et le plan continu de la toile elle-même. À partir du moment où la rencontre esthétique n'est plus un acte rétrospectif d'imagination mais un événement de perception immédiat, alors «la peinture ne signifie plus la mnémotechnie» (ibid., Georges Braque : 263).

L'analyse du cubisme livrée par Einstein dans ce texte, où il décrit le passage de la profondeur diaphane à la surface de la peinture, de la transparence sémiotique à la matérialité du signifiant, concorde à merveille avec les récits savants, pour nous familiers, qui présentent le modernisme comme une tendance esthétique privilégiant la présence matérielle de l'œuvre sur le contenu figuré. Depuis Roman Jakobson, nous savons que le réalisme incline à la transparence communicative, tandis que le modernisme valorise la fonction poétique et autoréférentielle du signe. Mais ce schéma se complique car, cinq ans après son article sur le cubisme analytique dans Documents, Einstein s'intéressa au cubisme synthétique tardif dans une étude des dernières œuvres que Braque venait de réaliser. Dans ce texte de 1934, Einstein concevait toujours l'entreprise cubiste comme une campagne menée contre la mnémotechnie du réalisme " descriptif "; cependant, les termes et la stratégie de l'offensive cubiste s'étaient considérablement modifiés, voire complètement inversés (fig. 3). À la différence des tableaux réalisés pendant la première phase cubiste, qui réduisaient par des procédés complexes la ressemblance entre l'image et son référent, les œuvres postanalytiques ne cherchaient plus à remettre aussi directement en cause la figuration et la ressemblance, mais faisaient au contraire appel à la figuration pour l'imprégner d'une profondeur énigmatique et hallucinatoire. Tandis que le cubisme analytique interdisait au spectateur l'accès psychologique à l'espace de l'illusion picturale, renvoyant celle-ci à l'espace plastique de la rencontre perceptive, les stratégies du cubisme synthétique tardif, telles que l'on peut les observer chez Braque à partir du début des années 1930, tendaient plutôt à renforcer cette illusion par leur indécidabilité et leur polyvalence sémiotique. Einstein insistait sur le fait que la traduction de motifs antiques dans le langage de l'écriture automatique surréaliste ne devait pas être confondue avec l'abstraction; elle représentait au contraire une forme de "réalisme mythique » (Einstein, Werke III : 301). La description et la ressemblance étaient de retour dans ces tableaux, même si les figures qui apparaissaient n'avaient rien à voir avec le modèle bien connu de la Gestalt intégrale des œuvres réalistes traditionnelles. Ces figures « métamorphiques », ainsi qu'Einstein les décrivait, gonflaient et s'interpénétraient. Le trait utilisé dans Héra et Thémis pour définir le contour de l'un des corps était par exemple incorporé à l'intérieur du personnage voisin, mettant ainsi en péril la silhouette de chaque figure. Ce tissage ininterrompu de lignes empêchait toute distinction, même la plus simple, entre les objets. Des lettres étaient elles aussi intégrées aux arabesques et projetaient les limites des figures dans l'espace textuel environnant. Dans ce champ «transvisuel», pour reprendre la formule d'Einstein, on ne pouvait plus dire ce qui formait une figure isolée, on ne savait plus ce qui constituait une figure distincte, et la perception la plus basique de la frontière entre l'objet situé dans le champ de vision et le fond était brouillée. 
Le fait que la méthode de Braque ait été fondamentalement additionnelle était un élément capital. Sa stratégie était de faire appel à la figuration pour ensuite la dépouiller de ses ressources en redessinant encore et encore les contours des formes individuelles, ce qui produisait ainsi une nouvelle définition du groupe figuré et créait une nouvelle composition à chaque boucle tracée d'un geste unique et continu. Le choix du médium de la gravure pour réaliser ces œuvres était significatif, puisqu'il est bien connu que cette technique ne pardonne aucune erreur. Les dessins peuvent être gommés et les toiles repeintes, mais il est virtuellement impossible d'effacer une ligne gravée. Les marques de la gravure sont fondamentalement indélébiles et le processus d'inscription est irréversible. Pourtant, Braque apportait la preuve que ces lignes pouvaient en réalité être révisées et transformées, si ce n'est qu'il fallait pour cela ajouter encore d'autres lignes. Cette exigence technique spécifique de la gravure exprimait un principe poétologique fondamental du cubisme postanalytique, qu'Einstein décrivait comme l'«augmentation (Vermehrung), l'accroissement de la forme (Gestaltzuwachs) et l'enchantement du réel» (ibid.: 324). Dans ces œuvres, Braque se contentait d'additionner et ne soustrayait jamais rien. Einstein appelait ce principe d'augmentation le "style double" et montrait que les tableaux de Braque représentaient simultanément des réalités picturales contradictoires et mutuellement exclusives. La stratégie employée différait radicalement de celle du cubisme analytique. Là où, jusqu'alors, ce dernier avait désactivé le code mimétique de la peinture réaliste en la fragmentant en différentes facettes, confrontant ainsi ses images-signes à l'opacité et au manque de relief à partir desquels elles devaient surgir de leur profondeur pour converger avec la surface de la toile, l'œuvre synthétique et postsynthétique ne cherchait plus à neutraliser la capacité signifiante de l'image. Au lieu d'être annulé, le symbole était désormais démultiplié, réécrit (umgedichtet) et métamorphosé (ibid.). Ces gravures ne niaient donc pas en bloc la représentation descriptive de la réalité, comme c'était le cas dans les premières œuvres de Braque, mais elles venaient au contraire compléter les objets dépeints jusqu'au point où ils devenaient quasi méconnaissables. La désactivation analytique était remplacée par une suractivation synthétique. À travers ces figures polyvalentes, le spectateur faisait l'expérience non pas de la négation de la référence, mais d'un surplus de référentialité qui générait un fil virtuellement infini d'analogies et de formes visuelles renvoyant continuellement les unes aux autres, en boucle.

Les deux articles d'Einstein sur le cubisme montrent que chaque génération de cubistes remit en cause le système du réalisme mimétique, bien que chacune ait mis en œuvre des stratégies opposées pour exécuter ce programme. À l'éradication de la trace mnémonique se substitua sa réinscription infinie. Cette transition poétologique, dans la pratique cubiste, entre des stratégies analytiques et des stratégies synthétiques, peut aussi être observée dans l'évolution d'Einstein lui-même comme écrivain, notamment dans le passage du modernisme originel de Bebuquin au Bebuquin II tardif. Quand Einstein écrivait sur le cubisme, il était le plus souvent en train de parler aussi, pour une part, de son propre travail littéraire. Dans sa fameuse lettre de 1923 à Daniel-Henry Kahnweiler, qu'il rédigea peu de temps après avoir commencé à travailler sur Bebuquin II, il expliquait que le cubisme était un phénomène culturel complexe dont la portée et les répercussions ne pouvaient être limitées aux arts visuels car, à un niveau épistémique plus profond, la critique de la mimèsis englobait toutes les formes de production symbolique, y compris la littérature ${ }^{4}$. Bien qu'Einstein ait évité de se prononcer

\footnotetext{
4. Dans cette lettre, Einstein écrivait : « Je sais depuis très longtemps que ce qu'on appelle "cubisme" dépasse de loin la peinture » (Correspondance Einstein/Kahnweiler, p. 48). II évoquait ensuite certains aspects de la littérature cubiste. À la fin de la lettre, Einstein disait à nouveau : «Je ne crois pas que le cubisme soit seulement une spécialité optique; s'il l'était, il serait faux, car non fondé. Expérience valable, il englobe beaucoup plus et je crois que pour le comprendre, ce n'est qu'une question d'énergie. » (Ibid., p. 57)
} 


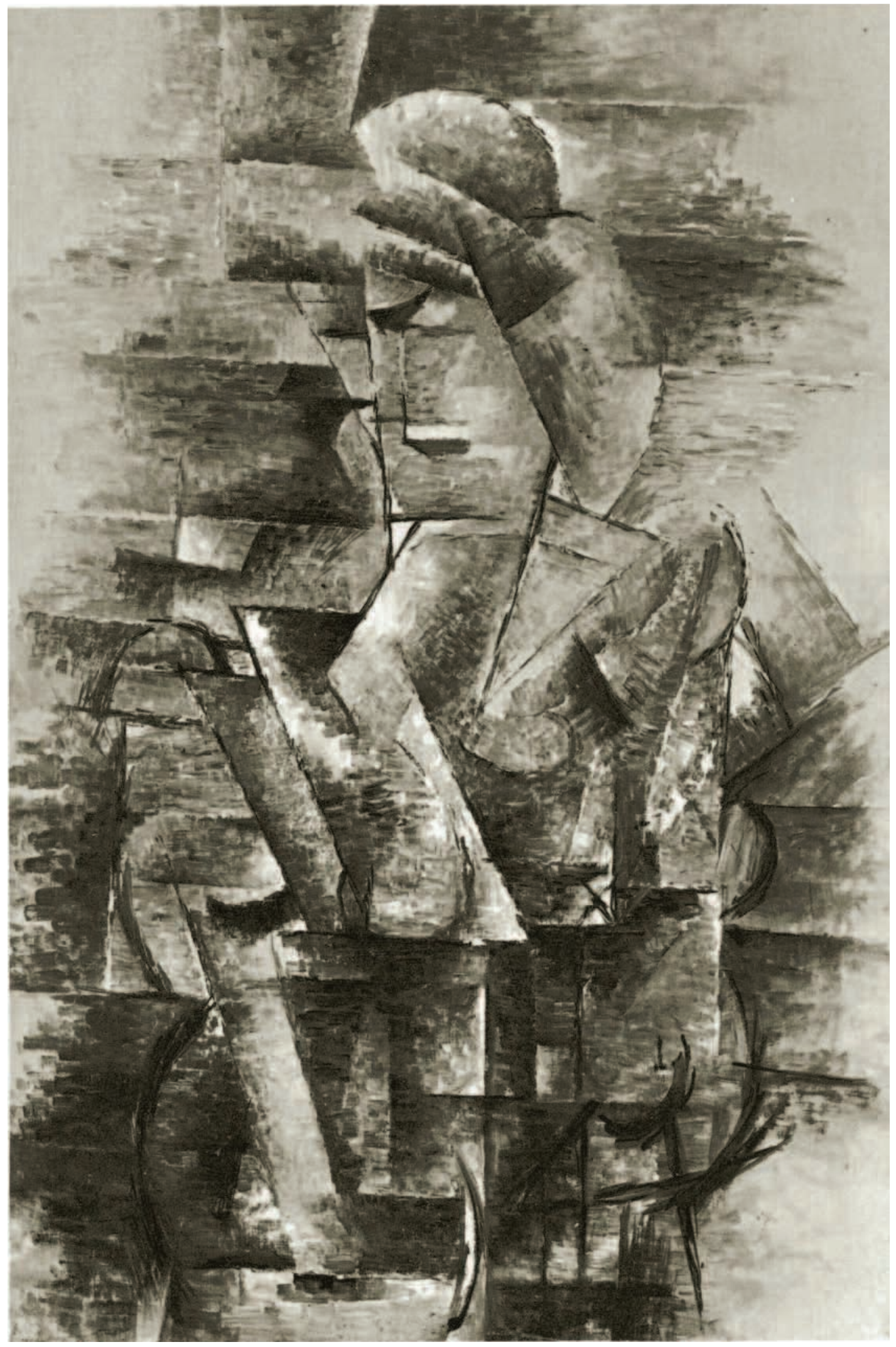

Fig. 4 Georges Braque, Torse, 1910 @ ADAGP, Paris 2011. 
sur une quelconque ressemblance morphologique entre la peinture cubiste et une certaine littérature - il savait qu'une telle affirmation ne pourrait jamais être démontrée d'un point de vue formel, et qu'il en résulterait indéfiniment une recherche dans une série de métaphores douteuses -, il ne discernait pas moins certaines affinités entre les lois structurelles d'énonciation à l'œuvre dans les deux médiums. Il est légitime de tracer un parallèle entre les deux phases de la peinture cubiste et les deux projets Bebuquin d'Einstein car, à chaque étape, les œuvres sont historiquement contemporaines : tout comme Bebuquin, rédigé en 1906, reflète le type analytique du cubisme qui émergeait alors à la même époque, Bebuquin II, sur lequel Einstein se mit à travailler au milieu des années 1920, épouse les principes artistiques du cubisme synthétique tardif qu'Einstein découvrait dans les dernières œuvres réalisées à l'époque par Braque.

À l'instar du cubisme analytique, Bebuquin orientait le lecteur vers le jeu des signes qui se déroulait à la surface du texte, où, par exemple, des schémas de rimes phonétiques dictaient la séquence des mots. De telles stratégies avaient pour but de saper la profondeur mnémonique et la densité de la représentation, d'annuler l'image mentale du signifié et, en retrouvant la valeur acoustique pure du langage, de provoquer chez le sujet le même état de présence perceptive et de grâce que le cubisme analytique recherchait à travers la pratique d'une peinture comme valeur optique pure. Bebuquin II s'efforçait de provoquer le même état d'oubli psychologique, mais il y parvenait par des stratégies d'excès de représentation, comme Braque à partir des années 1920. Ce texte, qui entassait les images mnémoniques les unes sur les autres, créait entre ces strates des ponts de ressemblance qui brouillaient les contours distincts des figures. À ce titre, l'« effort désespéré pour se débarrasser de la mémoire séculaire " (Archives Carl Einstein 8) que constituait Bebuquin II faisait écho au premier programme du cubisme analytique, mais l'amnésie n'était cette fois pas atteinte en éradiquant la mémoire et son stock d'imagerie mentale; paradoxalement, elle était obtenue par une augmentation métastatique et une accumulation infinie de figures trouvées dans les profondeurs de l'esprit.

Bebuquin II suivait une loi de l'économie mnémonique qui est au fondement de tout processus signifiant. Dans un essai sur l'«art de l'oubli », Umberto Eco extrapole cette loi à partir d'un fait, le caractère fondamentalement indélébile de la mémoire-trace. Eco remarque que, une fois inscrits, les souvenirs ne peuvent être effacés : «Toute expression déterminée par une fonction de signe sémiotique met en jeu une réponse mentale dès qu'elle est énoncée et empêche alors d'utiliser une autre expression pour faire disparaître son propre contenu. Si les arts de la mémoire sont des sémiotiques, il est inconcevable de construire sur leur modèle un art de l'oubli5... » Parce que, inévitablement, les signes évoqueront toujours d'autres signes, note Eco, ils ne peuvent servir à faire disparaître les signes. Ainsi que l'avait observé Abélard, l'énoncé Nulla rosa est ne manquera jamais de faire apparaître à l'esprit une rose ${ }^{6}$. Ou, pour citer un exemple emprunté à l'œuvre d'Einstein : Eine Verteidigung des Wirklichen (Une défense du réel) reste Eine Verteidigung des Wirklichen. Même le signe ainsi nié, barré, continue de postuler l'existence de son référent. Eco conclut que, pour cette raison, il n'existe pas de méthode active permettant d'oublier la mémoire-trace qui connecte le signifiant à un concept correspondant. En dépit de l'impossibilité fondamentale d'éradiquer l'inscription mnémonique, Eco insiste sur le fait que les structures de la mémoire peuvent néanmoins être affaiblies par la saturation. La mémoiretrace qui relie le signifiant et le concept ne peut être brisée, mais elle peut être

\section{- -1}

5. Umberto Eco, «An Ars Oblivionalis? Forget It ! », trad. Marilyn Migiel, $P M L A$, vol. CIII, 3, 1988, p. 259.

6. Ibid., p. 258. 
détournée, brouillée, perturbée. Pour Eco, il est possible « d'oublier non par défaut mais par excès, tout comme il est possible, non d'annuler le sens d'un énoncé prononcé à haute voix, mais de prononcer un autre énoncé au même moment, de telle sorte qu'ils se superposent. Il n'existe pas d'instrument volontaire de l'oubli, mais il existe des techniques pour se souvenir mal : il faut multiplier la sémiosis. " En l'absence de technique sémiotique permettant de provoquer activement l'amnésie, les signes peuvent toujours être déployés de manière à perturber et saper la mémoire. Dès lors, Eco conclut : «On oublie non par annulation mais par superposition, non en produisant de l'absence mais en multipliant les présences7. "

C'est là la stratégie déployée par Einstein dans son autobiographie, qui, pénétrant dans un état d'oubli par la petite porte, pour ainsi dire, s'efforce d'oublier par l'entremise d'une pratique incessante de remémoration. À chaque itération, l'image-souvenir précédente est recouverte et inscrite dans de nouveaux champs d'association qui orientent différemment la chaîne référentielle. Les conséquences de ce processus sur la fonctionnalité de la représentation symbolique sont peut-être encore plus désastreuses que ne le fut le tournant phénoménologique du modernisme esthétique pour la facture et la matérialité du signifiant. En "multipliant les présences", comme le suggère Eco, Bebuquin II entasse signe sur signe, s'effondre sur lui-même, et l'ordre signifiant tout entier subit un processus de désémantisation. Einstein définit les opérations mnémoniques étranges de son autobiographie comme « un souvenir du moi porté à son point d'anéantissement " (Archives Carl Einstein 14).

Ces stratégies de répétition et de réinscription se retrouvent dans Bebuquin II à des niveaux textuels multiples. D'abord, de la façon la plus évidente, elles se manifestent au niveau des dramatis personae de l'ouvrage : Einstein crée tout un jeu de ressemblances qui brouillent les frontières entre les figures individuelles. Le personnage principal de l'autobiographie est parfois évoqué à la troisième personne - Beb ou Laurenz -, mais, à d'autres moments, Einstein construit le récit depuis la perspective de la première personne. Il redistribue sans cesse les rôles. Dans certaines notes de travail, il se demandait s'il ne fallait pas faire naître Laurenz «de la métamorphose de quelqu'un d'autre» (Archives Carl Einstein 8). On comprend alors les références récurrentes à la réincarnation dans les notes du projet. Les personnages individuels n'ont ni point d'origine distinct ni destin personnel propre et s'engendrent les uns les autres à travers une série de transformations actantielles. Ce processus de recodage continuel rappelle la stratégie qu'Einstein nommait, en référence à Braque, l'«accroissement de la forme». À chaque accumulation additionnelle, à chaque transfert d'attributs et échange de rôles, les contours des personnages individuels se brouillent un peu plus.

Même une œuvre intitulée Bebuquin II ne pouvait réussir à contenir et à encadrer de telles proliférations. Après tout, l'autobiographie d'Einstein n'était pas une création ex nihilo, mais le retour à un texte de 1912 qu'il venait compléter et écraser simultanément. Construit sur le texte moderniste, Bebuquin II le révoquait du même coup. Tout comme Laurenz venait à la vie " par la métamorphose de quelqu'un d'autre", Bebuquin II émergea de la métamorphose de Bebuquin. C'est pourquoi, en dépit d'efforts textologiques impressionnants, les archivistes et les spécialistes d'Einstein n'ont toujours pas réussi à dessiner de manière convaincante les frontières de Bebuquin II. Le projet n'est structurellement conforme à aucun modèle textuel particulier et ne peut même, en toute rigueur, être rangé sous aucun type de discours, que ce soit la théorie, la fiction ou le documentaire. Le prototype textuel de ces 1300 unités d'écriture n'est semble-t-il pas 
le livre relié, mais plutôt le catalogue de fiches, une stratégie d'organisation du savoir qui repousse en permanence toute conclusion systématique. Comme le fait remarquer Denis Hollier dans un article sur l'usage de la fiche chez Michel Leiris, un tel «système de classement est extensible à l'infini et rhizomatique (à n'importe quel moment et à n'importe quel endroit, on peut insérer un nouvelle fiche); par contraste avec l'irréversibilité séquentielle des pages du cahier et du livre, sa mobilité intérieure autorise un réagencement constant ${ }^{8}$. " On ne sait pas exactement quand Einstein entreprit de transformer Bebuquin en Bebuquin II; Einstein ne déclara jamais non plus officiellement le texte terminé. Dans une lettre de 1923, peu de temps après avoir commencé la rédaction de Bebuquin II, il expliquait que ses propres mots lui paraissaient nécessairement inachevables. Le système textuel restait toujours ouvert à la révision : «Partout dans mes écrits j'ai le sentiment du fragment; toute ma vie, je pourrais sans cesse reprendre chaque phrase que j'ai écrite9. " De fait, des révisions furent apportées au projet jusqu'en 1940, et elles ne furent interrompues que par la mort d'Einstein.

La nécessité compulsive qu'éprouvait Einstein de revisiter et de réécrire son œuvre moderniste des débuts pour la transformer en une auto-anthologie correspond à une stratégie poétologique typique des années 1930. Ce procédé se retrouve à tous les niveaux de la production culturelle de l'époque : écrivains et artistes reprenaient leurs premières œuvres modernistes en les insérant dans un cadre autobiographique. Deux exemples issus du monde littéraire allemand sont particulièrement éclairants : Gottfried Benn, qui était l'ami d'Einstein, réécrivit ses légendaires Rönnenovellen (1916) sous la forme d'une autobiographie intitulée Lebensweg eines Intellektualisten (1934), et Walter Benjamin réutilisa bon nombre des «images de pensée" de son Sens unique (1926) dans son texte autobiographique plus tardif, Une enfance berlinoise ${ }^{10}$ (1938). On rencontre également ce type de réappropriation dans les arts visuels : au début des années 1930, Kazimir Malevitch, notamment, se lança de manière frénétique dans un projet récapitulatif de tous les développements du modernisme pictural depuis l'impressionnisme jusqu'au cubo-futurisme et au suprématisme; il ne se contenta pas de peindre à la manière de ces styles antérieurs mais, dans certains cas, recréa certaines œuvres. De la même manière, à la fin de la décennie, Marcel Duchamp réunit une collection de reproductions miniatures de ses œuvres d'avant-garde devenues des "classiques » dans un musée portable qu'il intitula, en référence au geste de double mise en boîte, La Boîte-en-valise.

C'est dans ce contexte culturel général, orienté vers des stratégies poétologiques de répétition et de recodage historique, qu'Einstein entreprit son projet d'anamnèse autobiographique, dont l'objectif n'était pas de reconstruire le passé mais de saturer le processus mémoriel. Sa stratégie en est un exemple : tandis que la répétition psychique pouvait servir un objectif de rétention et de régulation, la répétition multipliée à l'excès autorisait une expérience de nouveauté radicale ${ }^{11}$. Einstein expliquait ainsi que le moi adulte s'appuyait sur une réserve mnémonique gérable et ordonnée, une "petite pension " d'individualité que le sujet avait accumulée par un processus de "capitalisation psychique» (Einstein, Werke III : 97). Cependant, cette pension plaçait le moi au cœur d'une économie mentale régulée dans laquelle, nous l'avons vu plus haut, les choses étaient toujours transformées en signes et les expériences neuves servaient constamment à renvoyer aux souvenirs anciens. Afin d'échapper à la malédiction de la récurrence éternelle et de restaurer la possibilité d'un changement historique et d'une non-identité, Einstein voulait avoir recours à un état de conscience non encombré par la mémoire. Il écrivait:

\section{- $\bullet$}

8. Denis Hollier, « Notes (on the Index Card) », October 112, 2005, p. 40.

9. Lettre à Tony Simon-Wolfskehl, 25 janvier 1923. Citée dans Manuela Günter, Anatomie des Anti-Subjekts. Zur Subversion autobiographischen Schreibens bei Siegfried Kracauer, Walter Benjamin und Carl Einstein, Wurtzbourg, Königshausen und Neumann, 1996, p. 180.

10. Sur la pratique de l'autocitation et de la réécriture chez Walter Benjamin, voir Michael W. Jennings, «Double Take. Palimpsestic Writing and Image-Character in Benjamin's Late Prose », Benjamin-Studien 2, 2011, à paraître.

11. À ce titre, la pensée d'Einstein rejoint celle de Gilles Deleuze dans Différence et répétition, où Deleuze considère la notion de répétition comme une non-correspondance et une «différence sans concept »; Gilles Deleuze, Différence et répétition, Paris, PUF, 2000 [1969]. 


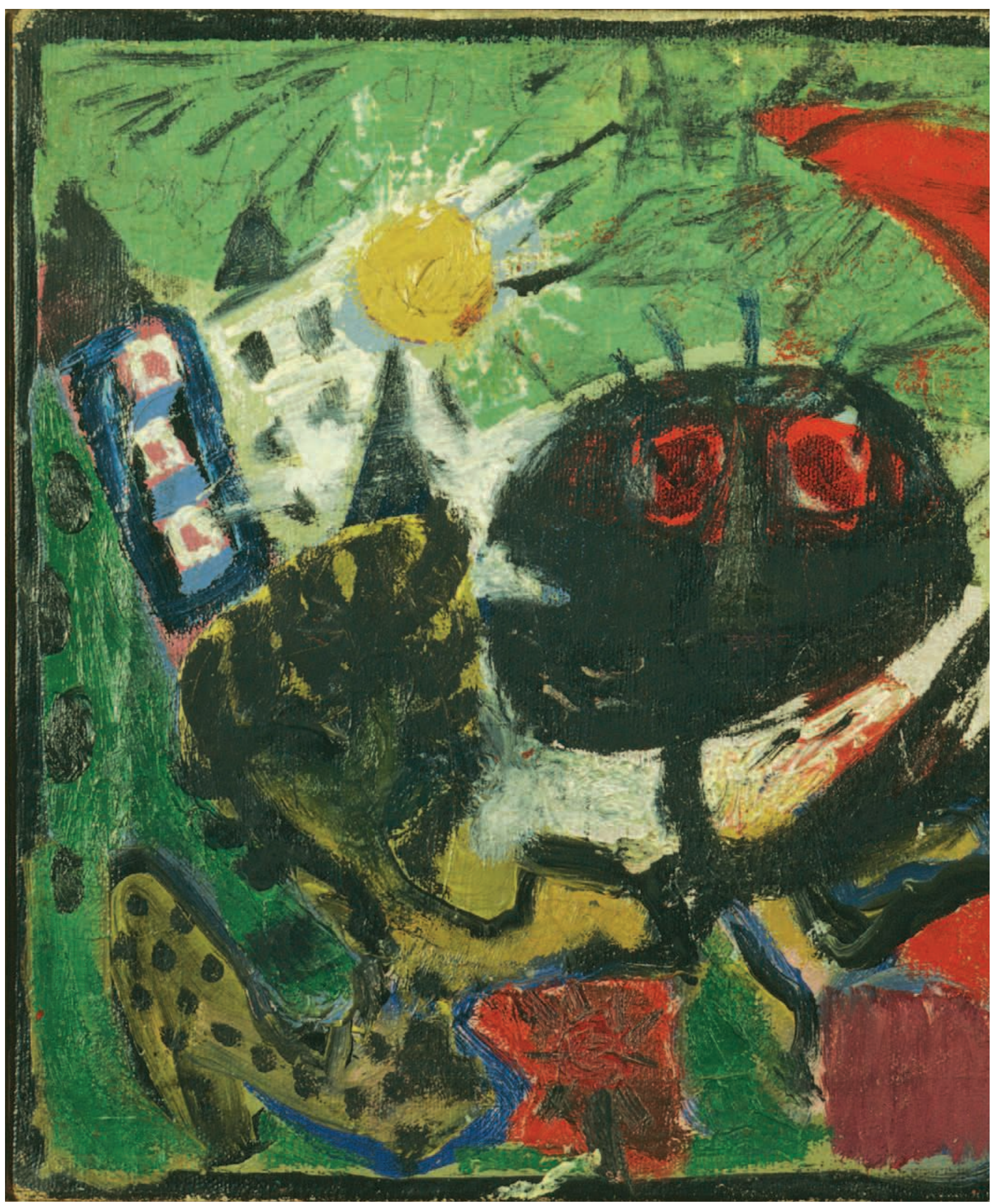

Fig. 5 Richard Mortensen avec la collaboration du groupe Cobra, Sans titre, 1949, don d'Asger Jorn 1959 au Museum Jorn / photo Lars Bay (c) ADAGP, Paris 2011. 


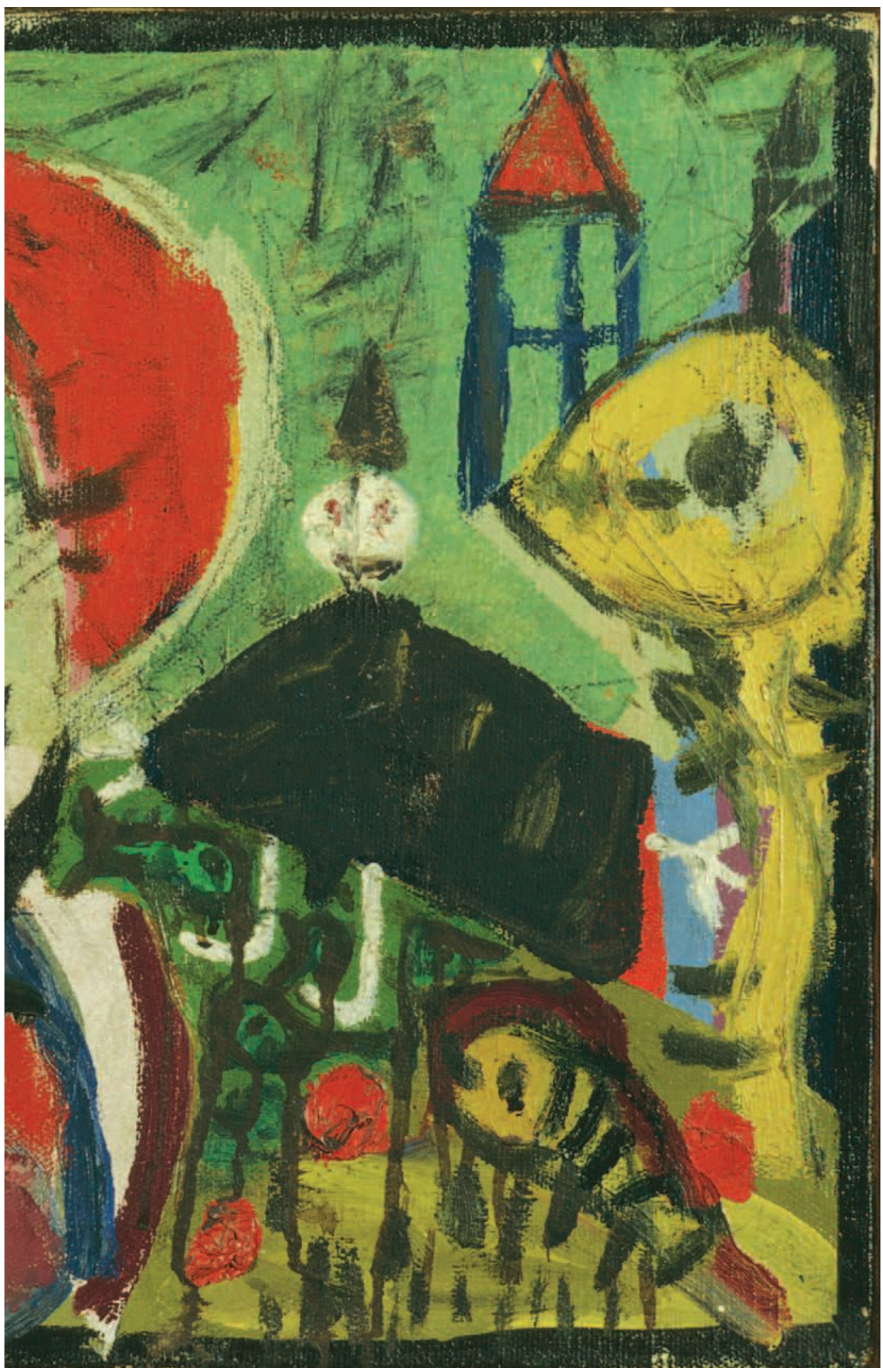




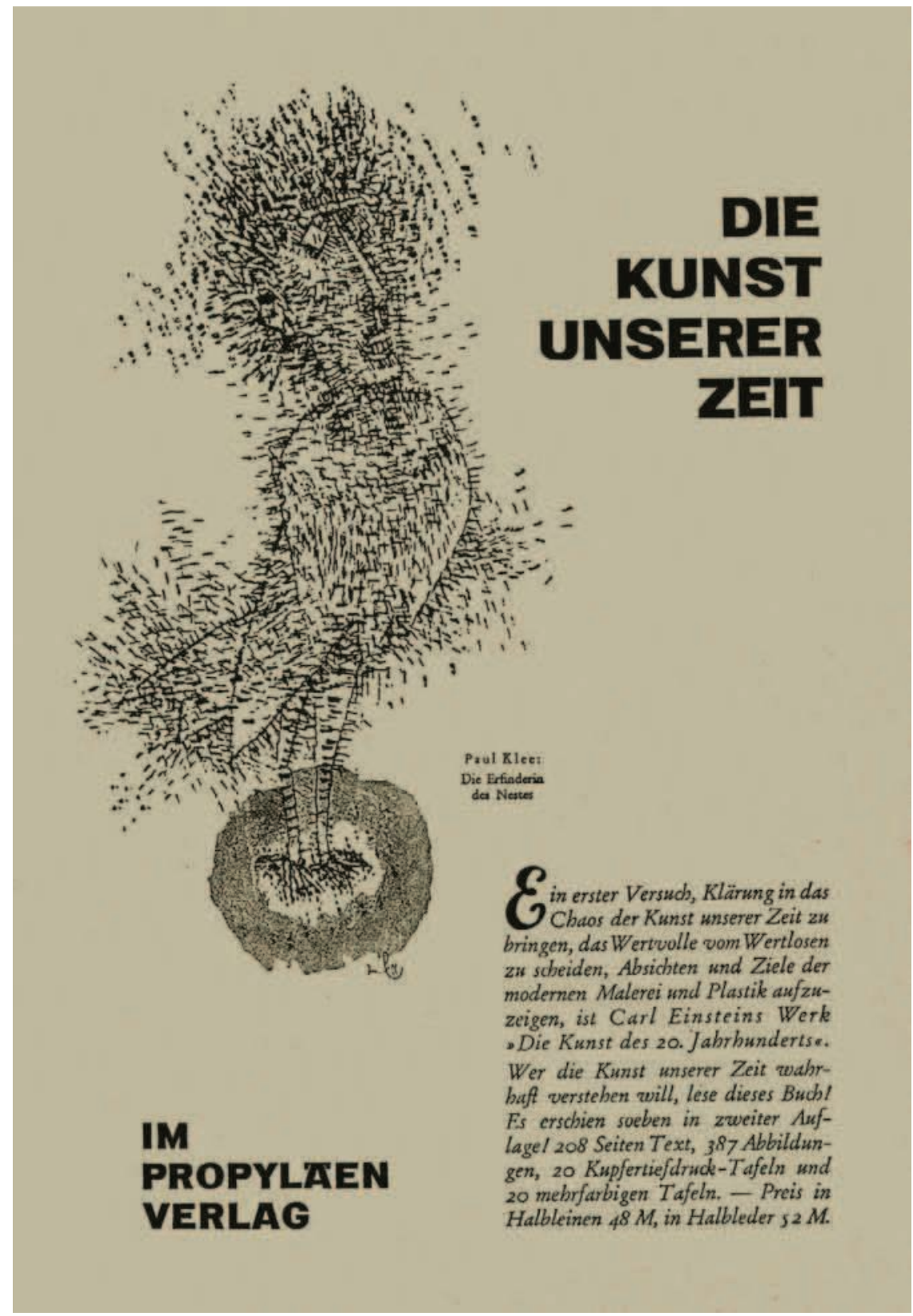

Fig. 6 Annonce pour la ze édition de Die Kunst des 20. Jahrhunderts de Carl Einstein (avec «Die Erfinderin des Nestes» de Paul Klee, 1925), parue dans Der Querschnitt, 1928.

"Tout ce qui est nouveau demande une extinction de la mémoire, c'est-à-dire de la conscience et du moi. » (Ibid. : 305) À l'instar de ses contemporains, de Viktor Chklovski à Benjamin, Einstein fit de la figure de l'enfant le paradigme de la conscience précatégorielle, du pur sujet phénoménologique libéré du poids de la coutume et des schèmes linguistiques incorporés. Parce qu'ils n'ont pas encore été entièrement initiés aux systèmes symboliques qui limitent le monde de la perception chez l'adulte en subordonnant les choses aux signes, les enfants «sont complètement enclins à l'anti-naturalisme» (ibid. : 292). Ils sont immunisés contre les effets de réel frauduleux de la représentation. 
Pour atteindre cet état de présence connu des enfants, et pour échapper à la prison du toujours-semblable, il était nécessaire, selon Einstein, de démanteler le système orthopédique mnémonique du moi adulte. Son autobiographie visait à rétablir cet état d'immédiateté de la perception en produisant un excès d'inscription mnémonique. À ce titre, elle semble avoir suivi une trajectoire exactement inverse de celle de la plupart des textes du même genre : à la différence des «documents du moi » autobiographiques typiques, qui rassemblent les expériences rudimentaires de l'enfance pour les incorporer dans un récit adulte et stable de l'individualité, Bebuquin II retournait à l'enfance non pour doter ces premiers moments ontogénétiques d'une structure et d'un sens, mais pour communier avec le chaos qu'ils formaient. Einstein disait que le telos de Bebuquin II était de « reconstituer l'enfance et de rendre tangibles ses origines par des régressions " (Archives Carl Einstein 31). La répétition de certains épisodes de son autobiographie devint un moyen de «retourner en arrière " (zurückblöden) vers son enfance (Archives Carl Einstein 29), vers la «zone sans mots» des «premières expériences en deçà du langage " (Archives Carl Einstein 7). Einstein qualifiait dès lors l'exercice autobiographique d' "entraînement à l'infantilisme " (Infantiltraining; Archives Carl Einstein 7). Il s'agissait de restaurer le mode de conscience qui existait avant que l'enfant n'ait été soumis aux schèmes de la grammaire, avant son passage par l'entreprise normalisatrice de l'éducation, avant que la pureté de perception n'ait été subordonnée aux conventions de la représentation - en résumé, avant son «idiotisation par l'intelligence» (Archives Carl Einstein 7). Cette économie mnémonique paradoxale expliquait à la fois «la permanence de la trace et la virginité de la substance d'accueil ${ }^{12} »$.

Face à la conception particulière que se faisait Einstein de l'entreprise autobiographique comme activité où l'inscription du moi coïncidait avec son effacement, on est ici tenté d'emprunter à Hegel une distinction terminologique qui aide à clarifier les mécanismes de la mémoire. Dans ses Cours d'esthétique, ce dernier soulignait le fait qu'il existe deux modalités fondamentalement différentes de la conscience rétrospective : Gedächtnis et Erinnerung, la mémoire et le souvenir. Bien qu'Einstein n'ait pas employé ces deux termes de manière aussi systématique que le philosophe de l'idéalisme allemand, la dynamique décrite par Hegel est essentielle pour saisir la conception einsteinienne de la mémoire. D'un côté, l'Erinnerung, une réserve dense et organique des expériences mentales à partir desquelles le sujet élabore son identité psychologique. Dans son commentaire des cours de Hegel, Paul de Man décrit l'Erinnerung comme un mode d'internalisation symbolique, «la réunion et la conservation intérieure de l'expérience ${ }^{13}$ ». À la différence de la remémoration, la mémoire, ou Gedächtnis, est une faculté presque mécanique qui, selon de Man, fonctionne par le moyen de la «notation », de l'«inscription" et de la "mise par écrit». Tandis que l'Erinnerung internalise l'expérience, la Gedächtnis inverse cette direction : elle objective les contenus de l'esprit et les projette sur le monde extérieur. Loin de développer notre capacité à nous souvenir, les aides et les techniques mnémoniques d'inscription qui sont au fondement de la Gedächtnis inhibent en réalité la vie mentale du sujet. Elles affaiblissent par exemple les aptitudes de l'imagination, la faculté qui gouverne l'agencement mental de la pensée symbolique. Ainsi, pour Hegel, un excès de mémoire, de Gedächtnis, n'entraîne pas une remémoration complète mais, bien au contraire, un oubli total. Par l'extériorisation de la mémoire, la lumière de la raison qui brûle dans l'esprit s'éteint et s'extériorise hors de la psyché du sujet. De Man résume cette logique déconstructiviste dans une formule qui rappelle de

\section{- $\bullet$}

12. Voir Jacques Derrida sur la convergence de la densité de l'inscription et de la stupeur béate dans le «bloc magique », ou le Wunderblock de Sigmund Freud; Jacques Derrida, «Freud et la scène de l'écriture », in L'Écriture et la différence, Paris, Seuil, 196?, p. 298.

13. Paul de Man, «Sign and Symbol in Hegel's Aesthetics », Critical Inquiry 8, 1982, p. 771 . 
-

14. Ibid., p. 773 .

15. Pour une analyse plus détaillée de cette célèbre anecdote, voir Harald Weinrich, Lethe. Kunst und Kritik des Vergessens, Munich, C.H. Beck, 1997, p. 92-105.

16. Paul de Man, «Sign and Symbol... », art. cit., p. 773.

17. Lévy-Bruhl décrit ainsi ce processus d'externalisation de la mémoire : «[L]a mémoire joue, dans la mentalité prélogique, un rôle plus considérable que dans notre vie mentale, où certaines fonctions qu'elle remplissait lui ont été enlevées et se sont transformées. [...] Les préliaisons, les préperceptions, les préraisonnements qui occupent tant de place dans la mentalité des sociétés inférieures n'impliquent point d'activité logique, et sont simplement confiés à la mémoire. II faut donc nous attendre à voir la mémoire extrêmement développée chez les primitifs. C'est en effet ce que les observateurs nous rapportent. » Lucien Lévy-Bruhl, Les Fonctions mentales dans les sociétés inférieures, Paris, Presses Universitaires de France, 1951 p. 116,11 ? manière surprenante la définition par Einstein de Bebuquin II comme «souvenir du moi porté à son point d'anéantissement » : "La mémoire efface la remémoration (ou le souvenir) tout comme le je s'efface lui-même ${ }^{14}$."

Un épisode antérieur de l'histoire de l'idéalisme allemand permet d'illustrer le processus qui, chez Hegel, relie la mémoire et l'extériorité à l'oubli, cette fois à partir d'une perspective davantage orientée vers les médiums techniques. L'histoire est la suivante. Dans les notes qui furent retrouvées dans les papiers de Kant après sa mort en 1804, l'une d'elles portait l'inscription suivante : «Le nom Lampe doit désormais tomber dans l'oubli complet. » Kant avait apparemment rédigé pour son propre compte ce petit pense-bête après une dispute avec son serviteur de longue date, Martin Lampe, qu'il démit de ses fonctions en 1802 après quarante ans de service. Pour écarter de son esprit la pensée pénible de Lampe, auquel Kant était bien entendu très attaché, le philosophe avait décidé de consigner sur le papier le souvenir de son serviteur. Il essayait ainsi, semble-t-il, d'oublier le nom de Lampe par son souvenir ${ }^{15}$. Une observation de De Man, toujours à propos de Hegel, peut livrer un commentaire pertinent de cette anecdote : " Pour avoir des souvenirs, on doit être capable d'oublier la mémoire et d'atteindre l'extériorité mécanique, de faire un détour par l'extérieur, ce que traduit l'expression allemande pour désigner l'apprentissage par cœur, aus-wendig lernen ${ }^{16}$." Deux siècles plus tard, Einstein, qui aspirait à atteindre cette condition d'«extériorité mécanique", reprit à son compte la stratégie kantienne de l'effacement, sauf que, cette fois, la mémoire qui devait être effacée mécaniquement n'était pas celle d'un compagnon mais celle de l'auteur lui-même.

Einstein désignait ce processus d'extériorisation comme une Veräußerung der Schrift (Archives Carl Einstein 7), expression qui traduit à la fois l'acte physique consistant à donner une forme matérielle à la mémoire, qui devient dès lors indépendante de son agent (une «extériorisation par l'écriture»), et l'expérience de défamiliarisation (estrangement) psychique et d'effacement du moi résultant de l'acte d'extériorisation mnémonique (une "aliénation par l'écriture»). Chacune des 1300 pages qui, dans ses valises, accompagnèrent Einstein dans son exil aliénait ainsi un autre moment de sa propre biographie. Bebuquin II révèle que l'entreprise autobiographique visant à confier les souvenirs à l'écriture, à l'archive, implique invariablement un double geste de commémoration et d'annihilation. Il s'agit d'une extériorisation qui met en péril la compétence de la mémoire organique et la faculté d'imagination. En outre, la validité de la loi mnémonique liant l'extériorisation à l'oubli n'est confirmée que par une formulation réciproque de cette même loi, dont l'anthropologie comparative nous dit qu'elle est également vraie: Lucien Lévy-Bruhl a montré dans Les Fonctions mentales dans les sociétés inférieures (1910), un ouvrage d'une grande importance dans l'évolution d'Einstein, que les membres des sociétés qui n'ont pas recours à la langue écrite pour transmettre le savoir possèdent des facultés de mémoire bien plus puissantes que les sociétés à écriture ou celles qui disposent d'autres technologies d'inscription ${ }^{17}$.

Dès lors, l'inscription technique n'entrave pas l'oubli mais permet et provoque même activement la progression vers un état d'oubli mental qui ressemble à celui de l'enfant. Bebuquin II fond ensemble les deux pôles de la tabula rasa de l'enfant et de la sénescence désordonnée de l'esprit âgé dans la figure oxymorique du Kindgreis, le "vieillard-enfant» (Archives Carl Einstein 14), qui devient le mandataire d'Einstein dans le texte. Einstein décrivait cet "entraînement à l'infantilisme " autobiographique comme la " quête d'une enfance perdue entre les feuilles et un 


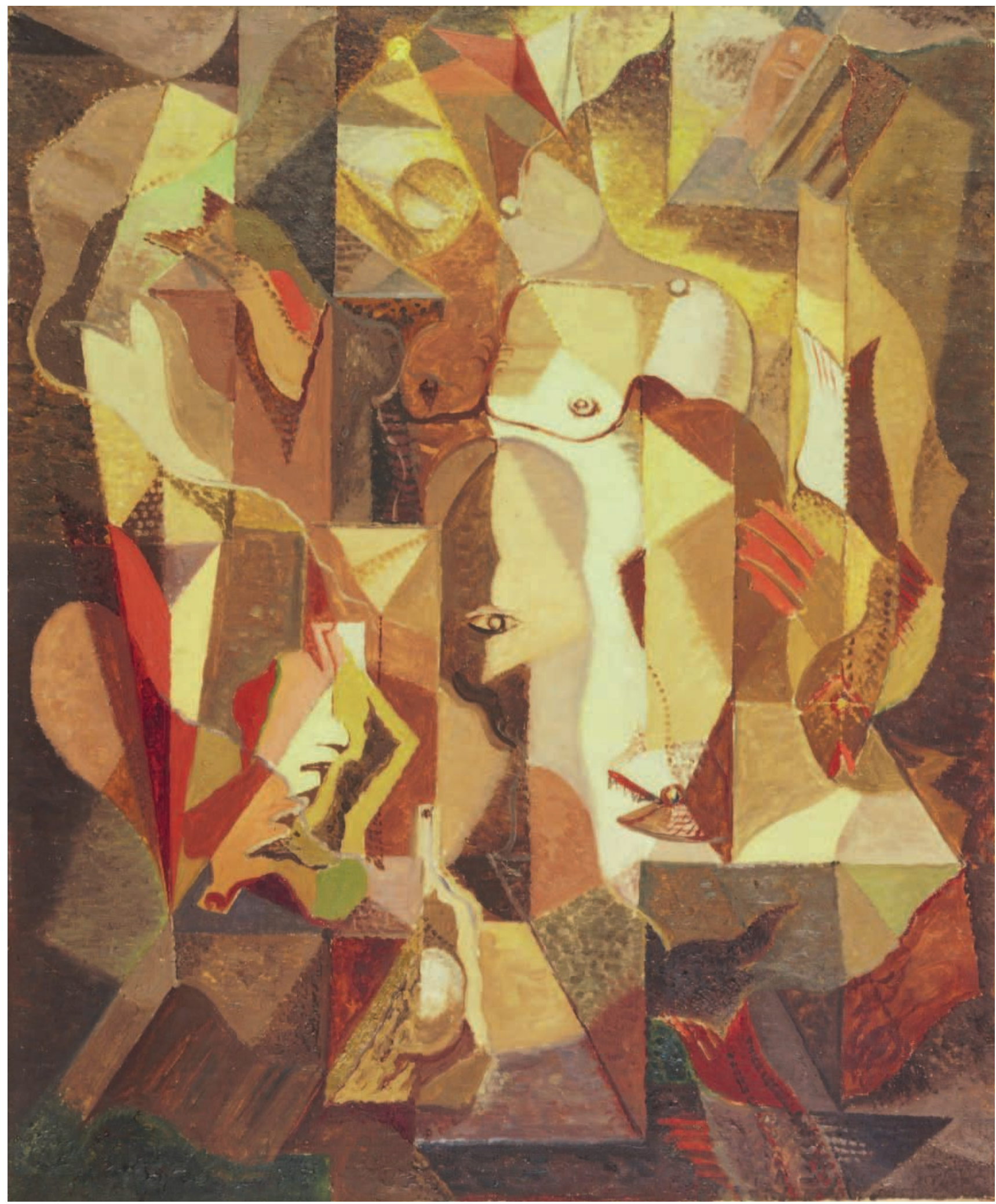

Fig. 7 André Masson, Le Bestiaire, 1925, musée d'Art et d'Histoire, Genève. 


\section{CARL EINSTEIN ET LES PRIMITIVISMES}

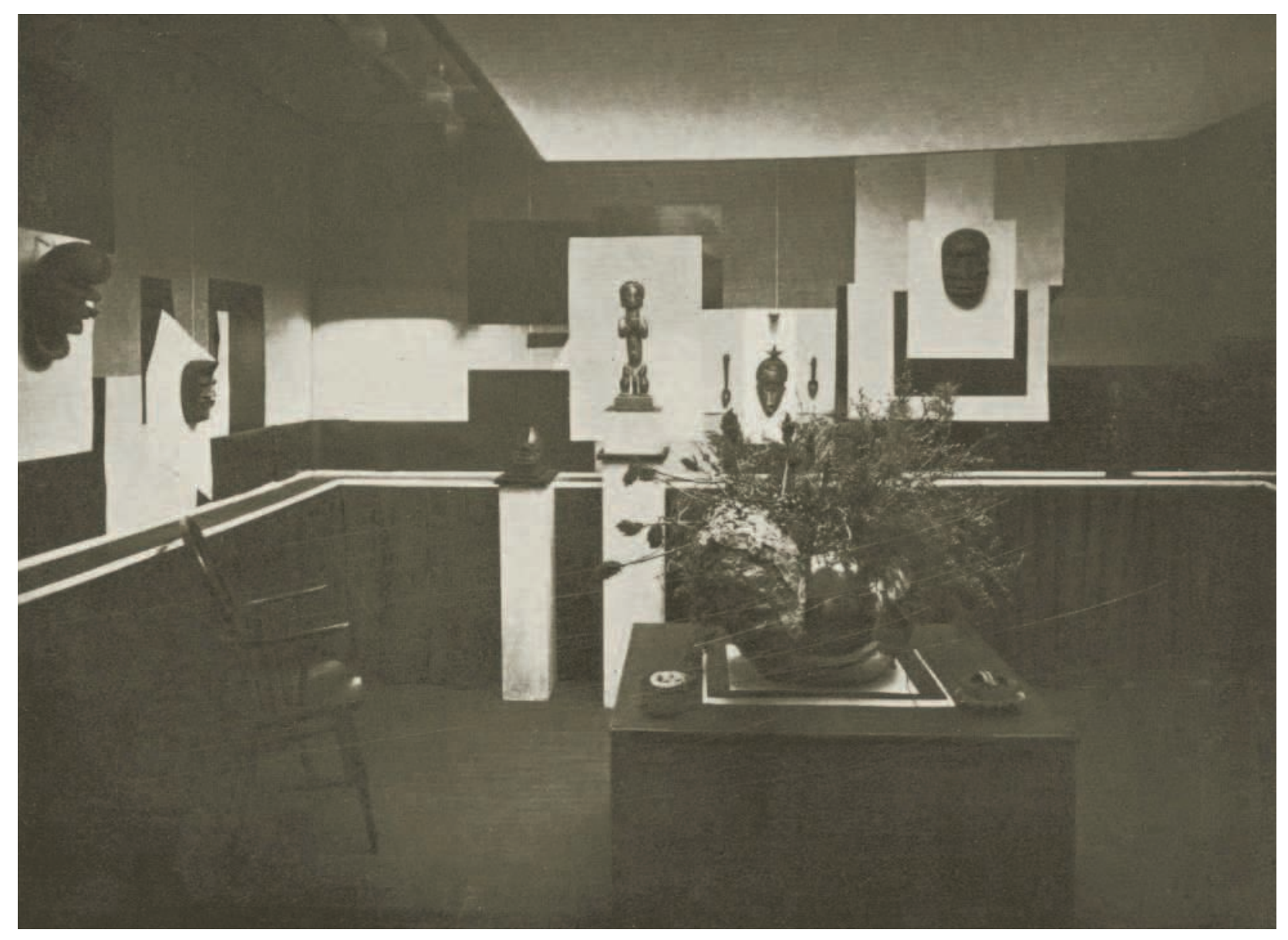

Fig. 8 Alfred Stieglitz, vue de l'expositon African Negro Art, MoMA, 1935, Paris, musée d'Orsay @ Georgia O'Keeffe Museum / ADAGP, Paris 2011 ○ RMN/René-Gabriel Ojéda.

oubli de ce qui a été appris" ("die suche nach der verlorenen kindheit zwischen blaettern und vergessen des erlernten"; Archives Carl Einstein 7). Le caractère polysémique de ces mots en dit long. D'un côté, les "feuilles ", blaetter, semblent être une référence spécifique à un épisode central de Bebuquin II qu'Einstein intitula le blaetterbegraebnis, l'« ensevelissement sous les feuilles ». Dans cette scène de sacrifice primitif, inspiré par la lecture de travaux d'anthropologues et d'ethnographes tel Lévy-Bruhl, les enfants se rassemblent dans la forêt pour étouffer à mort le prince enfant sous une montagne de feuilles. Comme dans d'autres passages du texte, Einstein reprend ici à son compte une figure typique de la pensée de l'époque, le parallèle entre le développement ontogénétique et phylogénétique associant les enfants non encore socialisés aux peuples dits «naturels ».

Dans le même temps, cependant, par-delà cette référence à un épisode particulier du récit diégétique, l'expression blaetterbegraebnis fait également figure de commentaire métapoétique sur le projet même de l'autobiographe, car ces blaetter ne sont pas seulement les feuilles qui tombent de l'arbre et recouvrent le sol. En allemand, blaetter désigne également les feuilles de papier. Le blaetterbegraebnis d'Einstein n'est pas simplement une référence au sacrifice du prince enfant de l'histoire mais aussi à la suffocation et à l'ensevelissement du moi de l'auteur sous les 1300 feuilles de matière biographique morte. De ce point de 
vue, la définition de Bebuquin II comme la «quête d'une enfance perdue entre les feuilles et un oubli de ce qui a été appris" renvoie à une autre dimension : l'important n'est plus de retrouver une origine authentique, mais d'atteindre, par l'acte même de la quête, un état de dissolution mentale. Si on traduit le mot blaetter par un verbe, blaettern - ce qui est ici permis car, comme dans presque tous les fragments tapuscrits de Bebuquin II, Einstein s'abstient d'utiliser des majuscules -, l'expression revêt un sens quelque peu différent, qui met l'accent sur le processus de l'enquête autobiographique : Bebuquin II devient alors une quête de l'enfance perdue par le procédé consistant à feuilleter les pages et, par cet acte, à oublier tout ce qui a été appris. Pour Einstein, l'autobiographie ne visait pas à arracher à la nébuleuse de sa jeunesse des épisodes spécifiques pour les mettre à l'abri, mais à reconstituer un mode de conscience propre à l'enfant par un processus d'extériorisation mnémonique.

Université de Princeton, département de langues et littératures slaves et département d'études germaniques dfore@princeton.edu

Traduction de l'anglais par Camille Joseph
PAGE 184

Fig. 9 Pablo Picasso, Tête, 1928, Paris, musée Picasso (c) RMN / Béatrice Hatala ๑) Succession Picasso 2011.

motsclés/keywords :Einstein, Carl // Einstein, Carl • autobiographie // autobiography - cubisme // cubism • anamnèse // amnesia • inscription // inscription.

\section{Résumé / Abstract}

Devin Fore, Un ensevelissement sous les feuilles : Bebuquin II, l'autobiographie de Carl Einstein - Cet article est consacré à Bebuquin II, l'autobiographie inachevée dont Carl Einstein entama la rédaction dans les années 1920. II s'agit de reprendre l'analyse livrée par Einstein du passage du cubisme analytique au cubisme synthétique et, à partir de là, d'examiner l'économie mnémonique singulière de Bebuquin II. De manière paradoxale, Einstein se donnait pour objectif, dans l'enquête autobiographique, la destruction du moi. II concevait Bebuquin /I comme une œuvre destinée à faire éclater les composantes de la subjectivité au lieu de les relier les unes aux autres, une anamnèse appelée à détruire le contenu de la mémoire par l'acte même de remémoration. L'éradication du noyau psychologique de l'individualité ne devait pas être obtenue par la négation du moi mais par un excès de moi et une surabondance de souvenirs.
Devin Fore, A Burial in Leaves: Bebuquin II, Carl Einstein's Autobiography - This essay focuses on the unfinished autobiography Bebuquin II, which Einstein began to write in the mid 1920s. Drawing upon Einstein's account of the transition from analytic to synthetic cubism, the essay examines the curious mnemonic economy of Bebuquin II, which seeks, paradoxically, to destroy the ego through autobiographical inquiry. Einstein envisioned Bebuquin II to be a work that would disperse the components of subjectivity rather than suturing them together, an anamnesis that would erase the contents of memory in the very act of their recollection. According to Einstein, the erasure of the psychological core of selfhood would be achieved not through the negation of the self, but through an excess of ego and a superabundance of memory. 